\title{
Dual Core Differential Pulsed Eddy Current Probe to Detect the Wall Thickness Variation in an Insulated Stainless Steel Pipe
}

\author{
C. S. Angani ${ }^{1,2}$, D. G. Park ${ }^{1 *}$, C. G. Kim² ${ }^{2}$ P. Kollu ${ }^{2}$, and Y. M. Cheong ${ }^{1}$ \\ ${ }^{1}$ Nuclear Materials Research Division, Korea Atomic Energy Research Institute, Daejeon 305-600, Korea \\ ${ }^{2}$ Department of Materials Science and Engineering, Chungnam National University, Daejeon 305-764, Korea
}

(Received 31 August 2010, Received in final form 30 September 2010, Accepted 4 October 2010)

\begin{abstract}
Local wall thinning in pipelines affects the structural integrity of industries like nuclear power plants (NPPs). In the present study, a pulsed eddy current (PEC) differential probe with two excitation coils and two Hall-sensors was fabricated to measure the wall thinning in insulated pipelines. A stainless steel test sample was prepared with a thickness that varied from $1 \mathrm{~mm}$ to $5 \mathrm{~mm}$ and was laminated by plastic insulation to simulate the pipelines in NPPs. The excitation coils in the probe were driven by a rectangular current pulse, the difference of signals from two Hall-sensors was measured as the resultant PEC signal. The peak value of the detected signal is used to describe the wall thinning. The peak value increased as the thickness of the test sample increased. The results were measured at different insulation thicknesses on the sample. Results show that the differential PEC probe has the potential to detect wall thinning in an insulated NPP pipelines.
\end{abstract}

Keywords : pulsed eddy current (PEC), differential probe, difference signal, insulation

\section{Introduction}

The Nondestructive testing (NDT) and evaluation of power plants and industrial systems are highly desirable due to safety and economic reasons $[1,2]$. Local wall thinning is an important point of concern because wall thinning can result in many leaks and causes damage to plant piping; it can also affect plant reliability and safety [3]. The pipelines are usually covered by thermal insulation for low thermal loss, so NDT methods that are capable of detecting wall thinning and defects without removing the insulation are necessary [4]. Pulsed eddy current (PEC) testing is an emerging technological NDT approach [5] which has the potential to detect the corrosion, as well as surface and sub-surface flaws [6]. There are several nondestructive and non-contact techniques, including the conventional eddy current (EC) technique [7] that uses a single frequency excitation; however, this technique has some limitations, including a limited depth of penetration or skin depth. The skin depth equation is given by $\delta=$ $\sqrt{1 / \pi \mu \sigma f}$ where $\mu$ is the permeability, $\sigma$ is the conductivity, and $f$ is the frequency of excitation, whereas pulse

*Corresponding author: Tel: +82-42-868-2023

Fax: +82-42-868-4785, e-mail: dgpark@kaeri.re.kr excitation (rectangular) was employed in PEC. The Fourier transform of a pulse train contains a series of frequency components [8], according to the skin effect different frequency components have different diffusion depths [9], hence the PEC technique has the potential to detect the defects at larger depths in a material.

For pipelines which are covered with insulation, it is necessary to detect the wall thinning under thicker insulations. To meet this requirement, the exciting magnetic field has to penetrate deeper. According to the analytical calculations of eddy currents [10] using the model of Dodd and Deeds [11], the coils with larger diameters which cover larger areas provide larger penetration depths [12]. To increase the field penetration, a dual core differential Hall-PEC probe was proposed in this study. Usually, the PEC probe consists of a driving coil and a detecting sensor. According to the Faraday's law of induction, a driving coil induces eddy currents in the test specimen. The induced eddy current fields were detected by the detecting sensor, but the detecting sensor detects not only the eddy current's field, but also the field due to the excitation coil. The exciting field is larger than the induced eddy current fields and hence the small changes due to perturbed eddy current fields due to flaws may easily be dominated by the excitation coil fields; to avoid this problem, there are 
two kinds of techniques that are used: one is reference signal subtraction $[13,14]$ and the other one is differential probes [15-17]. The differential probe method has the advantage of avoiding the storage of a reference signal before starting the measurements or testing.

\section{PEC System and Differential Probe}

The PEC system has a pulse generator which is connected to a pulse amplifier, a differential probe with two excitation coils and two Hall-sensors (HW-300), an amplifier to amplify the signal from the probe, a digital oscilloscope, an $\mathrm{A} / \mathrm{D}$ converter, and a computer with a data acquisition program. The probe was interfaced to the computer through a PCI (peripheral component interface) card for the data acquisition. The sensor probe consisted of two excitation copper coils with 150 turns wound on cylindrical ferrite cores with dimensions of $24 \mathrm{~mm}$ inner and $28 \mathrm{~mm}$ outer diameter. The two coils, which are wound on ferrite cores, are connected electrically in series and physically placed side by side with a small gap between them. Two Hallsensors (H1 and H2) are placed between the two excitation coils at top and bottom sides to detect the PEC response as shown in Fig. 1. The probe geometry and field distribution was simulated by using ANSOFT Maxwell [18] simulation software. Fig. 2 shows the simulated magnetic field distribution of the differential probe (simulated with sinusoidal excitation). The magnetic fields detected by the two sensors were subtracted using the difference amplifier (AD620) and the resultant signal was used as the probe signal. The calibration sample was a stainless steel (SS304) sample with a thickness ranging from $1 \mathrm{~mm}$ to $5 \mathrm{~mm}$ [19]. A plastic plate was laminated on the flat side of the sample to simulate the insulation of the pipelines as shown in the Fig. 1. A real time Lab-

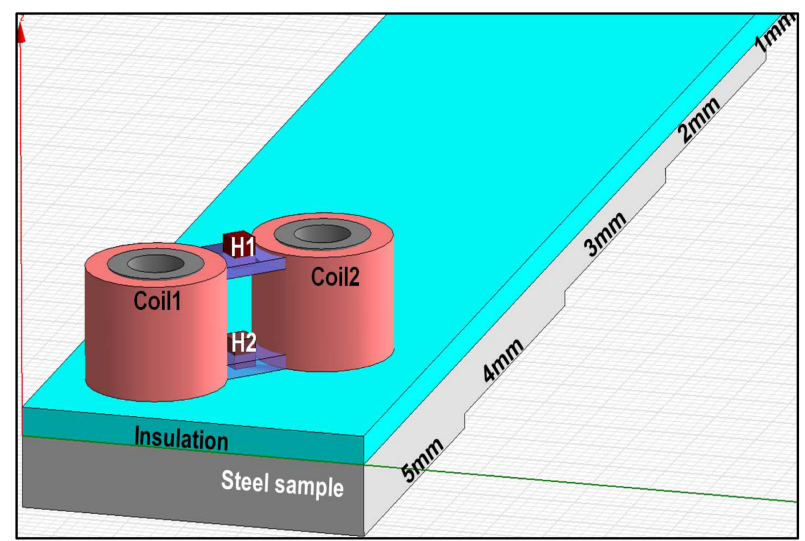

Fig. 1. Dual core differential PEC probe design and insulated steel sample.

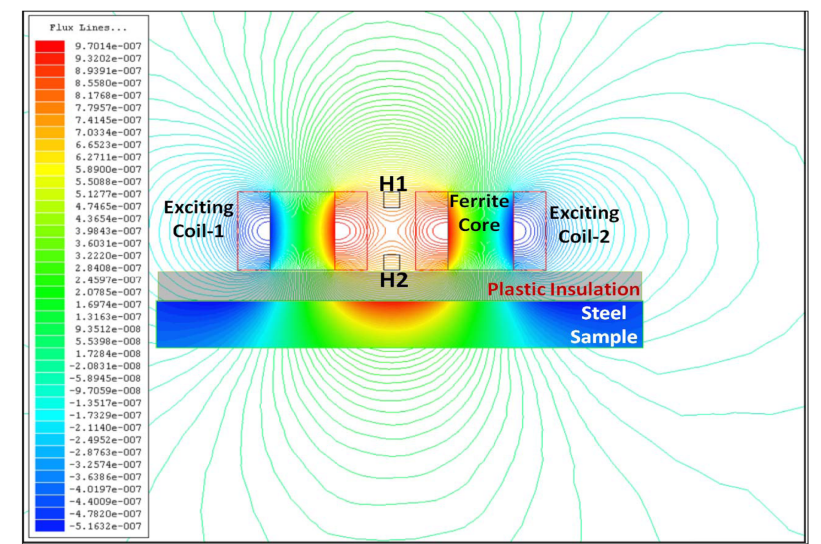

Fig. 2. The magnetic field distribution of a differential probe simulated with the ANSOFT Maxwell simulation software.

VIEW based data acquisition program was developed for scanning the probe on the insulated sample and the scanning results were continuously displayed on the computer monitor.

\section{Experimental Results and Discussion}

The excitation coil was driven by a rectangular pulse with $2 \mathrm{~ms}$ width and $1 \mathrm{~A}$ current. When we bring the probe into proximity of the conducting test sample, due to the pulse excitation, the responses from Hall-sensor1 (H1) and Hall-sensor2 (H2) can be explained as follows: the initial steeper part of the exciting pulse induces eddy currents in the test sample (higher rate of change of voltage induces eddy currents). The field of induced eddy currents counteracts the exciting coil's magnetic field, after that when the exciting pulse reaches the flat response (there is no rate of change in the exciting voltage), then the induced eddy currents exponentially decay to zero due to the electrical resistance of the specimen under test [20]. Hence, when the probe is placed on the conducting plate; the detected field rises slowly and exponentially to its steady state value [21]. Fig. 3 shows the response of individual Hall-sensors as well as the difference signal $\left(V_{\text {diff }}=H 1-\right.$ $H 2$ ) from the probe when it was placed on the $1 \mathrm{~mm}$ thickness of test sample. If there is an increase in the thickness of the test sample under the probe, the large cross sectional conduction area leads to higher induced eddy currents [22], hence the $\mathrm{H} 2$ (which is nearer to the sample surface) response rises slower than that of $\mathrm{H} 1$ to reach its maximum value [17]. As we are measuring the difference of the two sensors' responses, the difference signal $V_{\text {diff }}$ has an amplitude increase, so the differential pulse peak value increases as the specimen thickness increases.

The shape of the detected differential pulse is of interest 


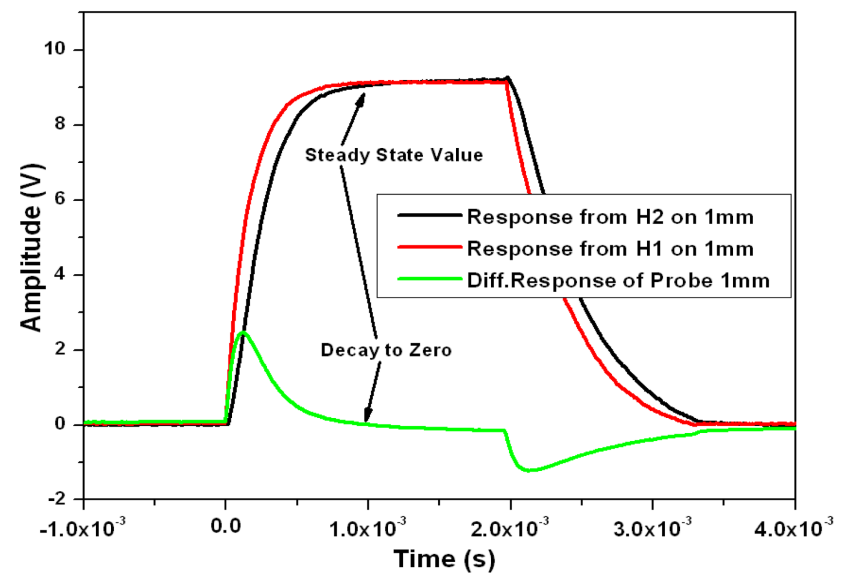

Fig. 3. Response from the individual Hall-sensors, $\mathrm{H} 1$ and $\mathrm{H} 2$, and differential signal $\left(V_{\text {diff }}\right)$ from the probe when it was mounted on the sample.

for the interpretation of results. Because the effect of the excitation field is nullified by the differential arrangement of the two Hall-sensors, only the induced eddy current fields are detected by the probe. Based on Fig. 3, we can understand $V_{\text {diff }}$ as a differential pulse response that is proportional to the induced eddy currents thus appeared initially in the transition region of the excitation pulse (according to Faraday's law, eddy currents induced during the transitions), so as the excitation pulse approaches its steady state value the induced eddy currents decay to zero, hence $V_{\text {diff }}$ approaches zero. Fig. 4 shows the results

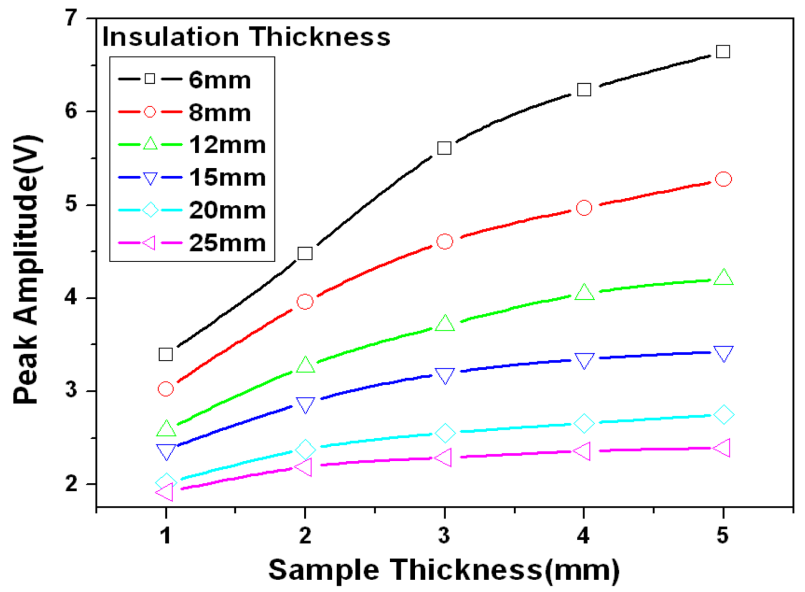

Fig. 5. The pulse amplitude as a function of sample thickness for different insulation thicknesses.
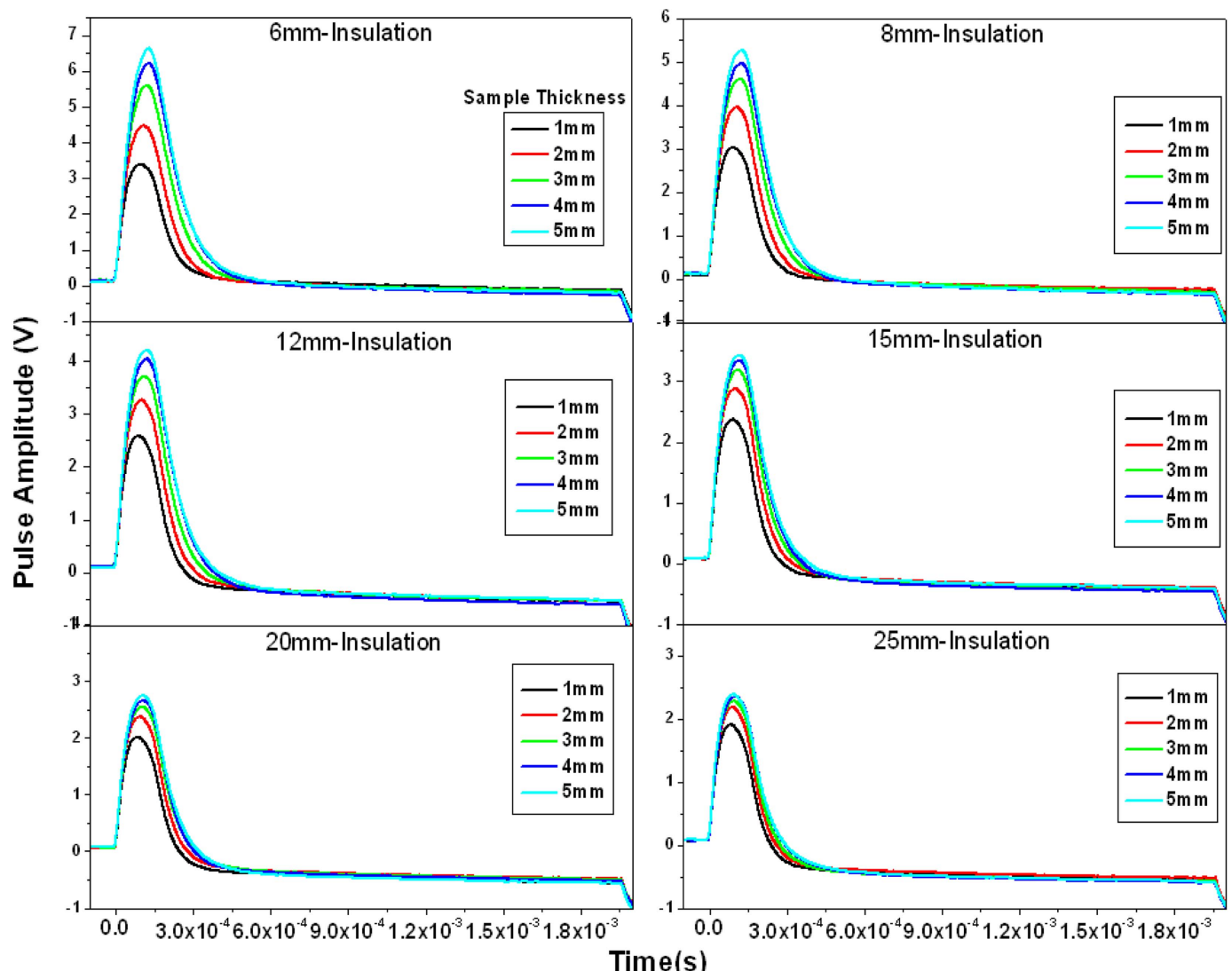

Fig. 4. Detected pulse response from different test sample thicknesses and at different insulation thicknesses. 


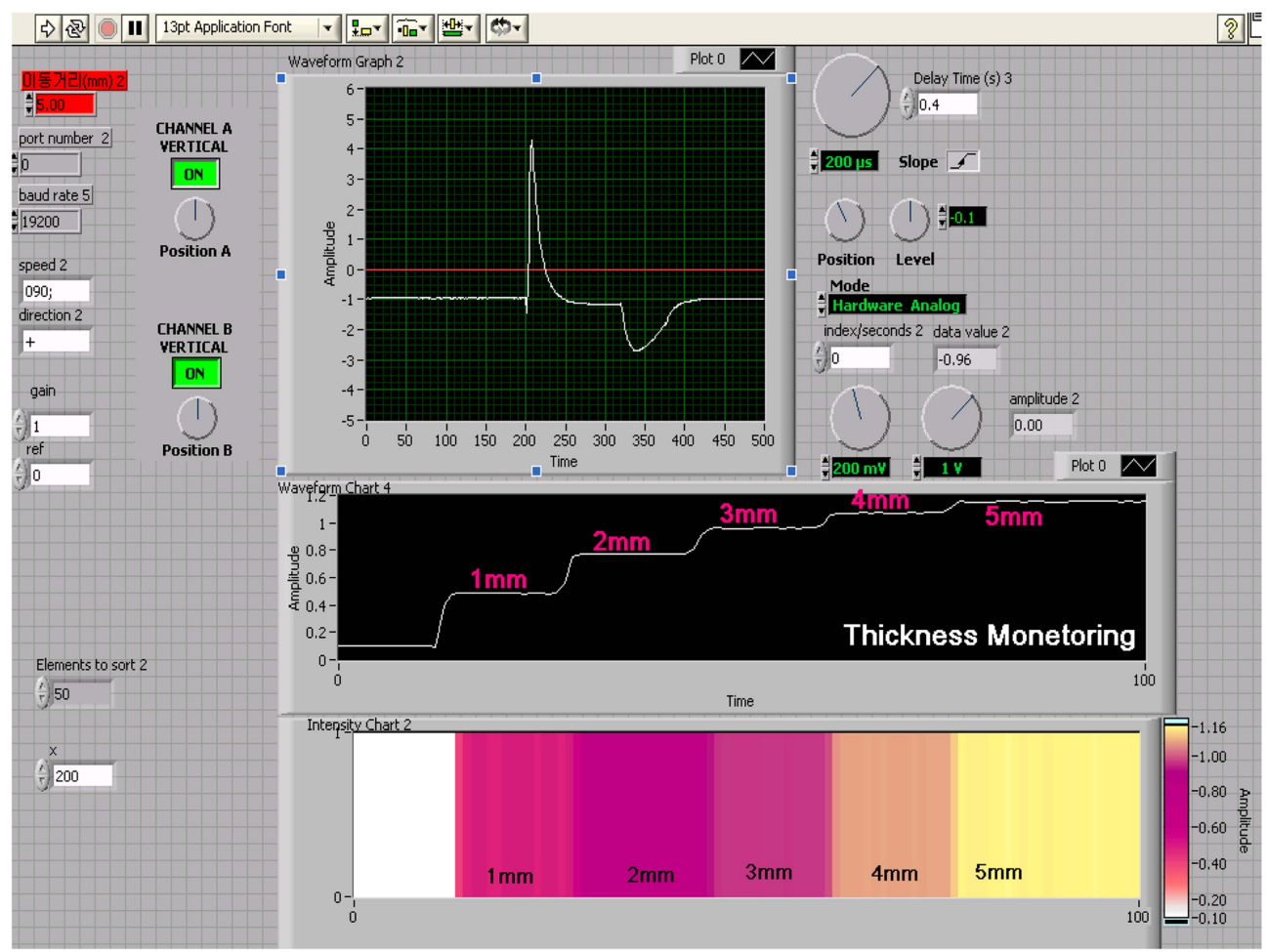

Fig. 6. The front panel controls of the LabVIEW based data acquisition program with an intensity chart.

which were measured at different insulation thicknesses on the tested sample; also, it was observed that the differential pulse amplitude increased with the thickness of the test sample. The detected pulse amplitude versus sample thickness for various insulation thicknesses that were obtained from Fig. 4 is shown in Fig. 5.

\section{Scanning of Test Sample}

A Lab VIEW based data acquisition program was developed to continuously monitor the variation in the thickness of the sample and was observed on the computer screen in a specified thickness monitoring window. Fig. 6 shows the front panel controls of the program. The time domain feature, which is the peak value of the detected pulse, is used for the scanning test to monitor the variation in the thickness of the tested sample. The PEC probe was scanned on the flat side of the tested sample which includes the plastic insulation. The intensity chart on the front panel shows the change in the thickness of the sample in terms of color variation according to the detected differential pulse amplitude.

\section{Conclusion}

A dual core differential probe which was used in a PEC system was fabricated for the detection of wall thinning in an insulated stainless steel pipe. The wall thinning of the calibration sample under different insulation thicknesses of up to $25 \mathrm{~mm}$ was investigated. The time domain feature, that is the pulse amplitude of the detected pulse, is used to understand the thickness variation; there is an increase of the detected pulse amplitude as the wall thickness of test sample increases because of the density of eddy currents which are induced in the tested sample. The scanning results were successfully displayed with an intensity chart on the computer monitor. The results show that the dual core differential PEC probe has the potential to detect wall thinning in insulated pipelines.

\section{Acknowledgement}

This work was developed by the research project focusing on the development of an advanced diagnostic technique for micro-material degradation and damages, as a part of a long-term nuclear R\&D program supported by the Ministry of Education, Science and Technology (MEST), Korea.

\section{References}

[1] J. Blitz, Electrical and Magnetic Methods of Nondestruc- 
tive Testing, Chapman \& Hall, London, (1997) p.163.

[2] G. Mustafa, Sensors and Actuators A 91, 70 (2001).

[3] K. M. Hwang, T. E. Jin, and S. H. Lee, Trans. 17th Int. Conf. Structural Mechanics Reactor Technology (Prague, Czech Rep.) paper O02-5, 7 (2003).

[4] M. A. Robers and R. Scottini, 8th ECNDT Conf. (Barcelona, Spain) NDT.net 7 (2002).

[5] B. C. Yoseph, Materials Evauation 58, 17 (2000).

[6] S. Giguere, B. A. Lepine, and J. M. S. Dubois, Research in Nondestructive Evaluation 13, 119 (2001).

[7] J. C. Moulder, E. Uzal, and J. H. Rose, Rev. Sci. Instrum. 63, 3455 (1992).

[8] I. Z. Abidin, C. Mandache, G. Y. Tian, and M. Morozov, NDT\&E Int. 42, 599 (2009).

[9] R. Griberg, L. Udpa, A. Savin, and R. Steigman, NDT\&E Int. 39, 264 (2006).

[10] Z. Motti, NDT Int. 23, 11 (1990).

[11] C. V. Dodd and W. E. Deeds, J. Appl. Phys. 39, 2829 (1968).

[12] M. Gerhard, H. Olaf, and U. Valentin, Proc. ECNDT
(Berlin, Germany) 5, 258 (2007).

[13] G. Y. Tian and A. Sophian, NDT\&E Int. 38, 77 (2005).

[14] B. Lebrun, Y. Jayet, and J. C. Baboux, NDT\&E Int. 30, 163 (1997).

[15] Y. K. Shin and D. M. Choi, NDT\&E Int. 42, 215 (2009).

[16] L. Shu, H. Songline, and Z. Wei, Sensors and Actuators A 135, 675 (2007).

[17] C. S. Angani, D. G. Park, G. D. Kim, and C. G. Kim, J. Appl. Phys. 107, 09E720 (2010).

[18] ANASOFT corporation, Maxwell EM V13, www.ansoft.com.

[19] D. G. Park, C. S. Angani, G. D. Kim, and C. G. Kim IEEE Trans. Magn. 45, 3893 (2009).

[20] J. Bowler and M. Johnson, IEEE Trans. Magn. 33, 2258 (1997).

[21] T. W. Krause, C. Mandache, and J. H. V. Lefebvr, Rev. Quantitative Nondest. Eval. 27, 368 (2008).

[22] W. Youhua, W. Junhua, L. Jiangui, and L. Haohua, Proc. Int. Conf. CTEEE (Novosibirsk, Russia) IEEE Region 8 Sibircon, 238 (2008). 